PROCEEDINGS OF THE

AMERICAN MATHEMATICAL SOCIETY

Volume 130, Number 2, Pages 443-451

S 0002-9939(01)06200-1

Article electronically published on June 6, 2001

\title{
A SYMMETRY THEOREM REVISITED
}

\author{
JOHN LEWIS AND ANDREW VOGEL
}

(Communicated by Juha M. Heinonen)

\begin{abstract}
We show that if harmonic measure and Hausdorff measure are equal on the boundary of certain domains in Euclidean $n$-space, then these domains are necessarily balls.
\end{abstract}

\section{INTRODUCTION}

Denote points in Euclidean $n$-space, $\mathbb{R}^{n}$, by $x=\left(x_{1}, \ldots, x_{n}\right)$, and let $\bar{E}$ and $\partial E$ denote the closure and boundary of $E \subseteq \mathbb{R}^{n}$, respectively. Put $B(x, r)=$ $\{y:|y-x|<r\}$ when $r>0$. Define $k$ dimensional Hausdorff measure, $1 \leq k \leq n$, in $\mathbb{R}^{n}$ as follows: For fixed $\delta>0$ and $E \subseteq \mathbb{R}^{n}$, let $L(\delta)=\left\{B\left(x_{i}, r_{i}\right)\right\}$ be such that $E \subseteq \bigcup B\left(x_{i}, r_{i}\right)$ and $0<r_{i}<\delta, i=1,2, \ldots$. Set

$$
\phi_{\delta}^{k}(E)=\inf _{L(\delta)}\left(\sum \alpha(k) r_{i}^{k}\right)
$$

where $\alpha(k)$ denotes the volume of the unit ball in $\mathbb{R}^{k}$. Then

$$
H^{k}(E)=\lim _{\delta \rightarrow 0} \phi_{\delta}^{k}(E), 1 \leq k \leq n .
$$

Let $D$ be a bounded domain in $\mathbb{R}^{n}$ with $0 \in D$ and $H^{n-1}(\partial D)<+\infty$. We assume that $D$ is a Dirichlet domain in the following sense: Given $h$ continuous on $\partial D$ there exists $H$ continuous in $\bar{D}$ and harmonic in $D$ with $H=h$ on $\partial D$. Under this assumption it follows from the Riesz representation theorem for continuous functions that there exists $\mu$ a positive Borel measure on $\partial D$ with $\mu(\partial D)=1$ and $H(0)=\int_{\partial D} h d \mu . \mu$ is called harmonic measure in $D$ with respect to 0 . We assume for some $r_{0}>0$ and $1 \leq L<\infty$ that $\mu$ satisfies the Ahlfors condition:

$$
\mu(B(x, r) \cap \partial D) \leq L r^{n-1} \text { for all } x \in \partial D \text { and } 0<r \leq r_{0} .
$$

In [LV92] we proved the following theorem.

Theorem A. Let $D$ be a bounded Dirichlet domain with $0 \in D$ and suppose that

(a) (1.1) holds for $\partial D$,

(b) $\mu=a H^{n-1}$ on $\partial D, a \equiv$ positive constant,

(c) $H^{n-1}\left(\partial D \backslash \partial D^{*}\right)=0$,

Received by the editors June 20, 2000.

1991 Mathematics Subject Classification. Primary 31B05, 31B20.

Key words and phrases. Harmonic measure, Hausdorff measure, quasiconformal, Green's function, Dirichlet problem.

The first author was supported in part by an NSF grant.

(C)2001 American Mathematical Society 
where

$$
\partial D^{*}=\left\{x \in \partial D: \limsup _{r \rightarrow 0} r^{-n} \min \left[H^{n}(B(x, r) \cap D), H^{n}(B(x, r) \backslash D)\right]>0\right\} .
$$

Then $D$ is a ball with center at 0 .

Actually in [LV92, Theorem 5] hypothesis (a) was replaced with the conclusion of Lemma 1 in section 2 . However given (b) both assumptions are equivalent as one proves easily using (2.1). In this paper we show that Theorem $\mathrm{A}$ can be improved upon. More specifically, we prove

Theorem 1. Theorem $A$ is valid under just hypotheses (a) and (b).

We note that Theorem 1 answers question (5) in [LV92, section 5]. We also note that (c) was used in a fundamental way in our proof of Theorem To see why, we briefly outline the proof of this theorem. Let $g\left(\cdot, x_{0}\right)$ denote Green's function for $D$ with pole at $x_{0} \in D$, normalized so that $\Delta g\left(x, x_{0}\right)=-\delta\left(x-x_{0}\right)$ in the distributional sense where $\Delta, \delta$ are the Laplacian and Kronecker $\delta$, respectively. If $u=g(\cdot, 0)$, then Theorem $\mathrm{A}$ follows easily from the following inequality using a barrier argument:

$$
\limsup _{y \rightarrow x}|\nabla u|(y) \leq a, \text { for all } x \in \partial D,
$$

where $a$ is the constant in (b) of Theorem A Indeed, choose $R>0$ so that $B(0, R) \subset D$ and $z \in \partial B(0, R) \cap \partial D$. Let $G$ be Green's function for $B(0, R)$ with pole at 0 . Then from the maximum principle we have $G \leq u$ in $B(0, R)$. Using this inequality, the mean value theorem from elementary calculus and the fact that $u, G$ extend continuously to $\bar{D} \backslash\{0\}$, it follows that

$$
\liminf _{y \rightarrow z}|\nabla G|(y) \leq \limsup _{y \rightarrow z}|\nabla u|(y) .
$$

Now $G$ is well known (see [Hel69]) and so by direct calculation we have

$$
\liminf _{y \rightarrow z}|\nabla G|(y)=\frac{1}{H^{n-1}(\partial B(0, R))} .
$$

Using (1.2) - (1.4), (b) of Theorem $\underline{\mathrm{A}}$, and the fact that $\mu(\partial D)=1$, we deduce that

$$
\frac{1}{H^{n-1}(\partial B(0, R))} \leq a=\frac{1}{H^{n-1}(\partial D)} \text {. }
$$

Thus

$$
H^{n-1}(\partial D) \leq H^{n-1}(\partial B(0, R)),
$$

which can only be true if $D=B(0, R)$ as we see from either the isoperimetric inequality or the fact that Hausdorff measure decreases under a projection. Hence (1.2) implies Theorem $\mathrm{A}$

To prove (1.2) in LV92 we first observed from geometric measure theory arguments that (c) implies the existence of a measure theoretic normal $H^{n-1}$ almost everywhere. Using this fact and blowup type arguments we obtained that (1.2) holds nontangentially in certain cones $H^{n-1}$ almost everywhere. To complete the argument we then used subharmonicity of $|\nabla u|$ away from 0 and a Poisson integral type argument.

In the present paper we manage to show that (a) and (b) are enough to imply (1.2) without having to assume the existence of the cones mentioned above. In this respect our argument is in the spirit of [MMV96]. In fact Theorem 1 for an 
Ahlfors regular domain in $\mathbb{R}^{2}$ can be deduced from [MMV96] as we point out in a remark following Theorem 11. Our proof of Theorem 1 does not rely on the apriori knowledge of nontangential limits in cones and seems simpler to us than the proof in [LV92].

We note that Theorem 1 or even Theorem $\mathrm{A}$ implies that if $\partial D$ is the image of a sphere under a bilipschitz mapping of $\mathbb{R}^{n}$ and (b) holds, then $D$ is a ball. On the other hand we proved the following theorem in [LV] which answered a question in LV91.

Theorem B. Given $K>1$ there exists a $K$ quasiconformal mapping $g$ from $\mathbb{R}^{n}$ to $\mathbb{R}^{n}$ such that if $D=g(B(0,1))$, then $D \neq$ ball and $H^{n-1}=\mu$ on $\partial D$.

Similar examples in $\mathbb{R}^{2}$ were constructed earlier by [KL37. Recall that a function $g: \mathbb{R}^{n} \rightarrow \mathbb{R}^{n}$ is said to be $K \geq 1$ quasiconformal on $\mathbb{R}^{n}$ if:

(i) $g$ is a homeomorphism of $\mathbb{R}^{n}$ onto $\mathbb{R}^{n}$,

(ii) $g$ has distributional partial derivatives that are locally $n$th power integrable,

(iii) $\|D g(x)\|^{n} \leq K J_{g}(x)$ almost everywhere.

In (iii), $D g(x)=\left(\frac{\partial g_{i}(x)}{\partial x_{j}}\right)$ is the Jacobian matrix of $g$ and $\|D g(x)\|$ is the norm of $D g(x)$ as a linear operator on $\mathbb{R}^{n}$. Also $J_{g}(x)$ (the Jacobian of $g$ at $x$ ) is the determinant of $D g(x)$.

Thus domains $D \neq$ ball for which $\mu=a H^{n-1}$ on $\partial D$ can be nice (quasiballs) but not too nice (bilipschitz images).

Finally, the authors would like to thank Juha Heinonen whose continued interest in this problem stimulated them to prove Theorem 1 .

\section{Several Lemmas}

In the proof of Theorem 1 we will need the following lemmas.

Lemma 1. Let $R>0$ be such that $B(0, R) \subset D, \partial B(0, R) \cap \partial D \neq \emptyset$. Then for some $N \geq L$, we have

$$
|\nabla u| \leq N<\infty \text { in } D \backslash B(0, R / 4)
$$

Here $u$ is Green's function for $D$ with pole at 0 .

Proof. To prove Lemma 1 we use the following formula obtained from the Riesz representation formula for subharmonic functions (see [Hel69]):

$$
\int_{0}^{r} t^{2-n} \nu(B(x, t)) \frac{d t}{t}=\int_{S} V(x+r \omega) d H^{n-1} \omega=m(x, r, V),
$$

where $S$ is the unit sphere in $\mathbb{R}^{n}$ and $V$ is subharmonic in $B(x, 2 r)$ with Riesz mass $\nu$ and $V(x)=0$. To use (2.1) we first extend $u$ to a subharmonic function in $\mathbb{R}^{n} \backslash\{0\}$ by putting $u \equiv 0$ in $\mathbb{R}^{n} \backslash D$. Applying (2.1) with $V=u, \nu=\mu$, using (1.1) and well known estimates for subharmonic functions we conclude for $x \in \partial D$ that

$$
\max _{B(x, r / 2)} u \leq c m(x, r, u) \leq c r
$$

when $0 \notin B(x, 2 r)$ and $r \leq r_{0}$. Here $c$ as in the sequel denotes a positive constant $\geq 1$ depending only on 'the data', not necessarily the same at each occurrence. In general we write $c\left(a_{1}, a_{2}, \ldots, a_{n}\right)$ when we wish to indicate dependence on $a_{1}, \ldots, a_{n}$. 
Let $d(x)$ denote the distance from $x$ to $\partial D$. Using (2.2) and the Poisson integral formula for harmonic functions we deduce that

$$
|\nabla u|(x) \leq c u(x) / d(x) \leq c \text { for } x \in D \cap\left\{y: d(y)<r_{1} / 8\right\},
$$

where $r_{1}=\min \left\{r_{0}, d(0)\right\}$. From (2.3) and the maximum principle for harmonic functions we conclude that Lemma 1 is valid.

Next we have

Lemma 2. For $H^{n-1}$ a.e. $x \in \partial D$,

$$
\limsup _{r \rightarrow 0} \frac{H^{n-1}(B(x, r) \cap \partial D)}{\alpha(n-1) r^{n-1}} \leq 1 .
$$

Proof. Lemma2 follows easily from the definition of Hausdorff measure (see Mat95. Ch. 6] where a different normalization is used).

We note for fixed $j, 1 \leq j \leq n$, that if $V(x)=\max \left\{x_{j}, 0\right\}$, then $\nu(B(0, t))=$ $\alpha(n-1) t^{n-1}$ so from (2.1) with $x=0$ we get

$$
\alpha(n-1)=\int_{S \cap\left\{\omega_{j}>0\right\}} \omega_{j} d H^{n-1} \omega .
$$

To prove (1.2) and thus Theorem 1 we put

$$
\inf _{\delta>0} \sup \{|\nabla u(y)|: d(y) \leq \delta\}=\limsup _{x \rightarrow \partial D}|\nabla u|=M
$$

and shall show that

$$
M \leq a .
$$

To this end assume $M>a$, let $\epsilon>0, \epsilon<10^{-10} a$ and let $x_{0} \in D \backslash B(0,3 R / 4)$ with

$$
\left|\nabla u\left(x_{0}\right)\right| \geq M-\epsilon
$$

Let $W(x)=\max \{|\nabla u(x)|-(M-2 \epsilon), 0\}$ and observe that $W\left(x_{0}\right)=\epsilon$. Choose $t>0$, so that $\left|\nabla g\left(\cdot, x_{0}\right)\right| \neq 0$ on $\left\{x: g\left(x, x_{0}\right)=t\right\}$ and $B(0,3 R / 4) \subset\left\{x: g\left(x, x_{0}\right)>t\right\}$. This choice is possible by Sard's theorem. Since $W$ is subharmonic in $D \backslash B(0, R / 2)$ we have

$$
\epsilon \leq W\left(x_{0}\right) \leq \int_{\left\{g\left(\cdot, x_{0}\right)=t\right\}} W(y) \frac{\partial g}{\partial n_{y}}\left(y, x_{0}\right) d H^{n-1} y+e\left(x_{0}, t\right)
$$

where $\frac{\partial}{\partial n_{y}}$ denotes the inner normal derivative at a boundary point $y$ of $\left\{g\left(\cdot, x_{0}\right)>\right.$ t\} and

$$
e\left(x_{0}, t\right) \leq c \int_{\partial B(0, R / 2)}\left(\left|\nabla_{y} g\left(y, x_{0}\right)\right|+\left|g\left(y, x_{0}\right)\right|\right) d H^{n-1} y
$$

where $c=c(N, R)$. Now in $D \backslash B(0, R / 2)$

$$
u(x) \leq c \frac{g\left(x, x_{0}\right)}{R^{n-2} u\left(x_{0}\right)}
$$

while in $D \backslash\left[B\left(x_{0}, d\left(x_{0}\right) / 2\right) \cup B(0, R / 4)\right]$ we have

$$
g\left(x, x_{0}\right) \leq c \min \left\{\frac{d\left(x_{0}\right)^{2-n}}{u\left(x_{0}\right)} u(x), \frac{d(x)^{2-n}}{u(x)} u\left(x_{0}\right)\right\},
$$


as follows from the maximum principle. Using Lemma 1 or (2.2) we see that

$$
|u(x)| \leq c d(x)
$$

when $x \in D \backslash B(0, R / 2)$.

Next from (2.8) with $x=y$, 2.9 with $x=x_{0}$ and gradient estimates as in (2.3), we find that

$$
e\left(x_{0}, t\right) \leq c d\left(x_{0}\right)
$$

where $c=c(N, R)$ is independent of $x_{0} \in D \backslash B(0, R / 2)$. From (2.10) we deduce that we may choose $x_{0}$ near enough $\partial D$ so that $e\left(x_{0}, t\right) \leq \epsilon / 2$. Thus if $t$ is so small that $|\nabla u| \leq M+\epsilon$ on $\left\{x: g\left(x, x_{0}\right)=t\right\}$, we have

$$
\epsilon / 2 \leq \int_{\{|\nabla u|>M-2 \epsilon\} \cap\left\{g\left(\cdot, x_{0}\right)=t\right\}} 3 \epsilon \frac{\partial g}{\partial n_{y}}\left(y, x_{0}\right) d H^{n-1} y
$$

and so

$$
\frac{1}{6} \leq \int_{\{|\nabla u|>M-2 \epsilon\} \cap\left\{g\left(\cdot, x_{0}\right)=t\right\}} \frac{\partial g}{\partial n_{y}}\left(y, x_{0}\right) d H^{n-1} y .
$$

Let $E(t)=\{x:|\nabla u|(x)>M-2 \epsilon\} \cap\left\{x: g\left(x, x_{0}\right)=t\right\}$. If $x \in E(t)$ or $x=x_{0}$, then we must have

$$
u(x) \geq M \frac{d(x)}{c}
$$

since otherwise it would follow from gradient estimates as in (2.3) that $|\nabla u|(x)<$ $M-2 \epsilon$. Let $x \in E(t)$ and consider the tangent ball $B(x, d(x))$. We see from (2.12) and (2.7)-(2.9) that $t=g\left(x, x_{0}\right) \leq c d\left(x_{0}\right)^{1-n} d(x)$ and $t=g\left(x, x_{0}\right) \geq c u\left(x_{0}\right) d(x)$. Thus for $c_{1}=c u\left(x_{0}\right), c_{2}=c d\left(x_{0}\right)^{1-n}$ we have

$$
c_{1} d(x) \leq t \leq c_{2} d(x) \text { for } x \in E(t)
$$

where $c_{i}=c_{i}\left(x_{0}, N, R\right), i=1,2$. We claim for $t>0$ sufficiently small that there exist tangent balls $\left\{B\left(x_{i}, d\left(x_{i}\right)\right)\right\}$, with $x_{i}=x_{i}(t) \in E(t)$ and

$$
\begin{gathered}
E(t) \subset \bigcup_{i} B\left(x_{i}, d\left(x_{i}\right) / 4\right), \\
B\left(x_{i}, d\left(x_{i}\right) / 100\right) \cap B\left(x_{j}, d\left(x_{j}\right) / 100\right)=\emptyset \text { for } i \neq j, \\
\sum_{i} d\left(x_{i}\right)^{n-1} \geq \frac{1}{c_{3}},
\end{gathered}
$$

where $c_{3}=c_{3}\left(x_{0}, N, R\right)$. In fact from (2.1) with $V=\max \left(g\left(\cdot, x_{0}\right)-t, 0\right)$,

$$
d \nu(y)=\frac{\partial g}{\partial n_{y}}\left(y, x_{0}\right) d H^{n-1} y .
$$

We see from (2.8), (2.9) as in (2.2) that for $x \in\left\{y: g\left(y, x_{0}\right)=t\right\}$

$$
\begin{aligned}
\int_{\left\{g\left(\cdot, x_{0}\right)=t\right\} \cap B(x, d(x) / 4)} \frac{\partial g}{\partial n_{y}}\left(y, x_{0}\right) d H^{n-1} y & \leq c\left(\max _{B(x, d(x) / 2)} V\right) d(x)^{n-2} \\
& \leq c d(x)^{n-1} .
\end{aligned}
$$


Next we use a well-known covering lemma to choose a cover of $E(t)$ by balls $\left\{B\left(x_{i}, d\left(x_{i}\right) / 4\right)\right\}$ with $\left\{B\left(x_{i}, d\left(x_{i}\right) / 100\right)\right\}$ pairwise disjoint:

$$
\begin{aligned}
\frac{1}{6} & \leq \int_{E(t)} \frac{\partial g}{\partial n_{y}}\left(y, x_{0}\right) d H^{n-1} y \leq \sum_{i} \int_{E(t) \cap B\left(x_{i}, d\left(x_{i}\right) / 4\right)} \frac{\partial g}{\partial n_{y}}\left(y, x_{0}\right) d H^{n-1} y \\
& \leq c \sum_{i} d\left(x_{i}\right)^{n-1} .
\end{aligned}
$$

Thus (2.14)-(2.16) are valid. From (2.13)-(2.16) we see for sufficiently small $t>0$ and $c=c\left(x_{0}, N, R\right)$ large enough that there are at least $\frac{t^{1-n}}{c}$ disjoint tangent balls with centers in $E(t)$. Finally in this section we prove

Lemma 3. With the above notation

$$
\begin{aligned}
& \int_{\left\{g\left(\cdot, x_{0}\right)>t\right\} \backslash B(0, R / 2)}\left[g\left(\cdot, x_{0}\right)-t\right] \Delta\left(|\nabla u|^{2}\right) d H^{n} y \\
& \leq\left. c \int_{\left\{g\left(\cdot, x_{0}\right)=t\right\}}|| \nabla u\right|^{2}-\left|\nabla u\left(x_{0}\right)\right|^{2} \mid \frac{\partial g}{\partial n_{y}}\left(\cdot, x_{0}\right) d H^{n-1} y+e^{*}\left(x_{0}, t\right)
\end{aligned}
$$

where $e^{*}\left(x_{0}, t\right) \leq c d\left(x_{0}\right)$ for some $c=c(N, R)$.

Proof. Use $g\left(\cdot, x_{0}\right)-t,|\nabla u|^{2}-\left|\nabla u\left(x_{0}\right)\right|^{2}$ in Green's second identity. $e^{*}\left(x_{0}, t\right)$ is estimated in the same way as $e\left(x_{0}, t\right)$. We omit the details.

\section{Proof of Theorem 1}

Proof. Using Lemma 1 and $\int_{\left\{g\left(\cdot, x_{0}\right)=t\right\}} \frac{\partial g}{\partial n_{y}}\left(y, x_{0}\right) d H^{n-1} y=1$ we see that the righthand side of Lemma 3 can be estimated by $c=c\left(x_{0}, N, R\right)$. Since $t$ is arbitrary we have

$$
\begin{aligned}
\int_{D \backslash B(0, R / 2)} g\left(\cdot, x_{0}\right) \Delta\left(|\nabla u|^{2}\right) d H^{n} y & \\
& =2 \sum_{i, j} \int_{D \backslash B(0, R / 2)} g\left(\cdot, x_{0}\right)\left(u_{x_{i} x_{j}}\right)^{2} d H^{n} y \leq c .
\end{aligned}
$$

Let $\delta>0, \delta \leq \epsilon^{100}$ be a small positive number to be chosen later. Put $\lambda=1-\delta$ and set $t_{k}=100^{-k}, k=1,2, \ldots$. From (3.1) it is easily seen for $k_{0} \geq 100$ a positive integer sufficiently large that

$$
\sum_{k=k_{0}}^{\infty} \sum_{j} \int_{B\left(x_{j}\left(t_{k}\right), \lambda d\left(x_{j}\right)\right)} d(x)\left|\nabla^{2} u\right|^{2}(x) d H^{n} x \leq c
$$

where $c=c\left(\delta, x_{0}, N, R\right)$. Indeed, using the fact that for fixed $k$ the balls $\left\{B\left(x_{j}\left(t_{k}\right), d\left(x_{j}\left(t_{k}\right)\right) / 100\right)\right\}$ are disjoint, it follows that each point in $D \backslash B(0, R / 2)$ lies in at most $c^{*}=c^{*}(\delta)$ of the balls $\left\{B\left(x_{j}\left(t_{k}\right), \lambda d\left(x_{j}\left(t_{k}\right)\right)\right)\right\}, k=k_{0}, \ldots$ Also by Harnack's inequality and (2.7), (2.12), we have $g\left(x, x_{0}\right) \geq \frac{d(x)}{c}$ for some $c=$ $c\left(\delta, x_{0}, N, R\right)$ in each of the balls. Using these facts in (3.1) we get (3.2). In (3.2) we 
can now use Poincaré's inequality and estimates for the oscillation of $u_{x_{j}}, 1 \leq j \leq n$, by way of the Poisson integral to get that if $\lambda_{1}=1-2 \delta$, then

$$
\sum_{k=k_{0}}^{\infty}\left(\sum_{j}\left(100^{-k}\right)^{n-1} \max _{x, y \in B\left(x_{j}\left(t_{k}\right), \lambda_{1} d\left(x_{j}\left(t_{k}\right)\right)\right)}|\nabla u(x)-\nabla u(y)|^{2}\right) \leq c
$$

for some $c=c\left(\delta, x_{0}, N, R\right)$. We claim there exists $k_{1} \geq k_{0}$ a positive integer such that whenever $k \geq k_{1}$ we have

$$
|\nabla u(x)-\nabla u(y)| \leq \delta
$$

on a subset $\left\{B\left(y_{j}, \lambda_{1} d\left(y_{j}\right)\right)\right\}$ of $\left\{B\left(x_{j}\left(t_{k}\right), \lambda_{1} d\left(x_{j}\left(t_{k}\right)\right)\right)\right\}$ ( $k$ fixed) with

$$
\sum_{j} d\left(y_{j}\right)^{n-1} \geq \frac{1}{\hat{c} c_{3}}
$$

where $\hat{c}=c\left(x_{0}, N, R\right)$ and $c_{3}$ is the constant in (2.16). Otherwise, from (3.3) and (2.13)- (2.15) we see that the inside sum for this $k$ is $\geq \frac{\delta^{2}}{c^{*}}$. Summing over $k$ we get a contradiction to (3.2). Thus (3.5) is true.

Next from Lemma 2 and real variable arguments we find that for given $\delta>0$ there exists $\rho=\rho(\delta)>0$ with

$$
\frac{H^{n-1}(B(z, r) \cap \partial D)}{\alpha(n-1) r^{n-1}} \leq 1+\delta, 0<r \leq \rho,
$$

for all $z \in \partial D$ except for a set of at most $H^{n-1}$ measure $<\delta^{100 n}$. We use (3.5) and (3.6) to show, for sufficiently small $\delta>0$, the existence of a positive integer $k_{2} \geq k_{1}$, such that whenever $k \geq k_{2}$ there exists $z=z(k) \in \partial D$ satisfying (3.6) and $z$ lies within $\delta 100^{-k}$ of a point of tangency of one of the balls $\left\{B\left(y_{j}\left(t_{k}\right), d\left(y_{j}\left(t_{k}\right)\right)\right)\right\}$. Indeed, assuming $\rho>>100^{-k_{2}}$ we see that if the above statement is false, then from (3.5), using disjointness of the balls $\left\{B\left(y_{j}, d\left(y_{j}\right) / 100\right)\right\}$ it would follow that there is a set contained in $\partial D$ with $H^{n-1}$ measure $\geq \frac{\delta^{n-1}}{c\left(x_{0}, N, R\right)}$ for which (3.6) fails. This is a contradiction if $\delta=\delta\left(x_{0}, N, R\right)>0$ is small enough.

We are now ready to prove Theorem 1 Let $z=z\left(k_{2}\right)$ be as above and let $B(y, d(y))$ be the guaranteed tangent ball corresponding to $k_{2}, z$. Let $\hat{z}$ be a point of tangency and observe that $\rho(\delta)>>d(y)$. From Lemma 1 we have for $0<r \leq \rho(\delta)$

$$
\begin{array}{r}
\int_{S} u(\hat{z}+r \omega) d H^{n-1} \omega \leq \int_{S} u(z+r \omega) d H^{n-1} \omega+c \delta d(y) \\
\leq a \alpha(n-1)(1+\delta) r+c \delta d(y)
\end{array}
$$

where $c$ is independent of $\delta$. To get the last inequality in (3.7) we used (b) of Theorem A] (2.1) with $V=u, \nu=\mu$ and (3.6). To estimate the left-hand side of (3.7) suppose $\hat{z}=y-d(y) \hat{e}_{n}$ where $\hat{e}_{n}=(0, \ldots, 0,1)$. On the one hand we deduce from (3.4) and $|\nabla u| \leq N$ that

$$
|u(x)-u(y)-\langle\nabla u(y), x-y\rangle| \leq c \delta d(y)
$$

for all $x \in \bar{B}(y, d(y))$ where $\langle\cdot, \cdot\rangle$ denotes the inner product in $\mathbb{R}^{n}$. On the other hand using this inequality for $\hat{z}$, (3.4), and $|\nabla u| \leq N$ we have

$$
\left|u(y)-\left\langle\nabla u(y), \hat{e}_{n}\right\rangle d(y)\right| \leq c \delta d(y) .
$$


Comparing these two estimates and using $u \geq 0$ we see that

$$
\left|\left\langle\nabla u(y), \hat{e}_{n}\right\rangle-\right| \nabla u|(y)| \leq c \delta
$$

where $c$ does not depend on $\delta$. Using (3.9) in (3.8) we find that

$$
\left|u(x)-\left\langle\nabla u(y), \hat{e}_{n}\right\rangle\left[x_{n}-y_{n}+d(y)\right]\right| \leq c \delta^{1 / 2} d(y) .
$$

Let $r=\delta^{1 / 4} d(y)$. For this value of $r$ we see from high school geometry that $\left[\left\{x: x_{n}-y_{n}+d(y) \geq 0\right\} \backslash B(y, d(y))\right] \cap \partial B(\hat{z}, r)$ has $H^{n-1}$ measure at most $c \delta^{1 / 4} r^{n-1}$ and $x_{n}-y_{n}+d(y) \leq c \delta^{1 / 4} r$ on this set. Using this observation, (3.10), and (2.4) we obtain

$$
\begin{aligned}
\int_{S} u(\hat{z}+r \omega) d H^{n-1} \omega \geq\left\langle\nabla u(y), \hat{e}_{n}\right\rangle r & \int_{S \cap\left\{\omega_{n}>0\right\}} \omega_{n} d w-c \delta^{1 / 4} r \\
& =\left\langle\nabla u(y), \hat{e}_{n}\right\rangle r \alpha(n-1)-c \delta^{1 / 4} r .
\end{aligned}
$$

Putting (3.11) in (3.7) we deduce that

$$
\left\langle\nabla u(y), \hat{e}_{n}\right\rangle \leq a+c \delta^{1 / 4}
$$

Using (3.12) and the fact that $|\nabla u(y)| \geq M-\epsilon$ we conclude from (3.9) that

$$
M-\epsilon \leq|\nabla u(y)| \leq\left\langle u(y), \hat{e}_{n}\right\rangle+c \delta \leq a+c \delta^{1 / 4} .
$$

Since $\delta$ can be arbitrarily small we have $M-\epsilon \leq a$. Letting $\epsilon \rightarrow 0$ we get $M \leq a$ which is a contradiction to our assumption that $M>a$. From this contradiction we first conclude (2.5) and then Theorem 1.

Remark 1. If $n=2$ it follows easily from MMV96 that Theorem 1 is true when $\partial D$ is Ahlfors regular. By definition $\partial D$ is Ahlfors regular if for some $r_{0}>0, K \geq 1$,

$$
K^{-1} r^{n-1} \leq H^{n-1}(B(x, r) \cap \partial D) \leq K r^{n-1}
$$

for all $x \in \partial D$ and $0<r \leq r_{0}$. We briefly outline the proof. From the Riesz representation theorem for subharmonic functions and (b) of Theorem $\mathrm{A}$ we deduce that

$$
2 \pi \nabla u(x)=-x /|x|^{2}+\int_{\partial D}(x-y)|x-y|^{-2} d H^{n-1} y \text { for } x \in D .
$$

The right-hand side of this equation is zero for $x \notin \bar{D}$. From Lemma 1 we see from this equality that

$$
\left|\int_{\partial D}(x-y)\right| x-\left.y\right|^{-2} d H^{1} y \mid \leq M_{1}<\infty
$$

whenever $x \notin \partial D \cup B(0, R / 4)$. Using this inequality and Ahlfors regularity it follows easily that

$$
\left|\int_{\partial D \cap\{|z-y|>\epsilon\}}(z-y)\right| z-\left.y\right|^{-2} d H^{1} y \mid \leq M_{2}<\infty
$$

for all $\epsilon>0$ and $z \in \partial D$. Let $r>0,0<r \leq R / 8$. Then (3.13) implies for given $r, z \in \partial D$, the existence of $\rho, r / 2 \leq \rho \leq r$ such that if $\chi=$ characteristic function of $B(z, \rho)$, then

$$
T_{\epsilon} \chi(x)=\left|\int_{\{|x-y|>\epsilon\} \cap \partial D} \chi(y)(x-y)\right| x-\left.y\right|^{-2} d H^{1} y \mid
$$


satisfies

$$
\int_{B(z, \rho) \cap \partial D}\left|T_{\epsilon} \chi\right|^{2} d H^{1} \leq c\left(M_{2}\right) r .
$$

In fact using Ahlfors regularity and (3.13) one first shows for $x \in \partial D$ that

$$
\left|T_{\epsilon} \chi\right|(x) \leq c\left(M_{2}\right) \log (\rho /|\rho-| x-z||) .
$$

Integrating this inequality in $x, \rho$ and using weak type estimates one gets (3.14). From (3.14) and Theorem[1] in [MMV96] we see that $\partial D$ is contained in a rectifiable curve. The argument in [LV92 can now be used essentially verbatim to get that $D$ = ball. Finally we note that since it is unknown whether Theorem 1 in [MMV96 has an analogue in $\mathbb{R}^{n}$ we were forced to come up with the simpler and more general proof of Theorem 1 above.

\section{REFERENCES}

[Hel69] L. Helms, Introduction to potential theory, Wiley-Interscience, 1969. MR 41:5638

[KL37] M. Keldysh and M. Lavrentiev, Sur la représentation conforme des domain limités par des courbes rectifiables, Ann. Sci. École Norm. Sup. 54 (1937), 1-38.

[LV] John L. Lewis and Andrew L. Vogel, On pseudospheres that are quasispheres, Rev. Mat. Iberoamericana, To appear.

[LV91] John L. Lewis and Andrew L. Vogel, On pseudospheres, Rev. Mat. Iberoamericana 7 (1991), 25-54. MR 92g:31006

[LV92] John L. Lewis and Andrew L. Vogel, On some almost everywhere symmetry theorems, Nonlinear diffusion equations and their equilibrium states, vol. 3, Birkhäuser, 1992, pp. 347-374. MR 93j:35078

[Mat95] P. Mattila, Geometry of sets and measures in euclidean spaces, Cambridge University Press, 1995. MR 96h:28006

[MMV96] P. Mattila, M. S. Melnikov, and J. Verdera, The Cauchy integral, analytic capacity, and uniform rectifiability, Ann. of Math. 144 (1996), 127-136. MR 97k:31004

Department of Mathematics, University of Kentucky, Lexington, Kentucky 405060027

E-mail address: john@ms.uky.edu

Department of Mathematics, Syracuse University, Syracuse, New York 13244

E-mail address: alvogel@syr.edu 